\title{
Warfarin Reinitiation After Intracranial Hemorrhage: A Case Series of Heart Valve Patients
}

\author{
Andrea Wan, Ken Butcher, Mathew Hodgson, Karen Schultz, Tammy J. Bungard (D)
}

\begin{abstract}
Patients with mechanical heart valves are at high thrombotic risk and require warfarin. Among those developing intracranial hemorrhage, limited data are available to guide clinicians with antithrombotic reinitiation. This 13-patient case series of warfarin-associated intracranial hemorrhages found the time to reinitiate antithrombotic therapy (17 days, interquartile range 21.5 days), and changes to international normalized ratio targets were variable and neither correlated with the type, location, or etiology of bleed, nor the valve and associated thromboembolic risk. The initial presentation significantly impacted prognosis, and diligent assessment and follow-up may support positive long-term outcomes.
\end{abstract}

RÉSUMÉ: Procurer une deuxième dose de warfarine à la suite d'une hémorragie intracrânienne : une étude de série de cas chez des patients à qui l'on a installé des valves cardiaques mécaniques. Les patients à qui l'on a installé une telle valve demeurent à risque élevé de thrombose et nécessite qu'on leur administre de la warfarine. Du côté de ceux ayant été victimes d'une hémorragie intracrânienne, peu de données sont disponibles afin d'orienter les cliniciens en ce qui concerne un traitement subséquent aux anti-thrombotiques. Cette étude de série de cas incluant 13 patients à qui l'on a administré de la warfarine à la suite d'une hémorragie intracrânienne a été en mesure de se baser sur un second traitement anti-thrombotique (17 jours ; EI 21,5 jours). Les changements par rapport aux cibles de rapport international normalisé (RIN) ont été variables et n'ont pas pu être corrélés au type, à l'emplacement ou à l'étiologie des saignements pas plus qu'au risque associé à la valve ou au risque thromboembolique. Chose certaine, cette étude initiale a eu un impact certain sur l'appréciation de l'évolution ultérieure de ces patients. En cela, il se pourrait qu'une évaluation et un suivi diligents puissent favoriser l'évolution à long terme de leur état de santé.

Keywords: Cardiology, Hemorrhage - Cerebral, Stroke

doi:10.1017/cjn.2019.331

Can J Neurol Sci. 2020; 47: 237-241

Patients with mechanical heart valves (MHVs) require chronic anticoagulation to reduce the risk of ischemic stroke and systemic thromboembolism. Warfarin is the only oral therapeutic option in this population, with poor outcomes associated with trials of dabigatran ${ }^{1}$ and dual antiplatelet therapy. ${ }^{2}$ Based on the valve location, the majority of North American patients with MHVs are targeted to an international normalized ratio (INR) range of either $2.0-3.0$ or $2.5-3.5$. The most feared complication of warfarin therapy is intracranial hemorrhage. Patients with MHVs who develop an intracranial hemorrhage while therapeutically anticoagulated require INR reversal to prevent further hematoma expansion. This poses a complicated clinical dilemma as clinicians must balance the risk of continued or rebleeding with the high thromboembolic risk associated with MHVs. At present, published data on post-intracranial hemorrhage antithrombotic management have focused on timing and strategies for INR reversal and the optimal time to reinitiate therapy. ${ }^{3,4}$ Guidance on INR targets post-intracranial hemorrhage is limited.

The Anticoagulation Management Service (AMS) in Alberta, Canada has provided antithrombotic management to over 4400 patients since its inception in 2001. From 2003 onwards, the AMS received direct referrals for all MHVs implanted in Edmonton and currently manages over 600 patients with MHVs.
We describe a case series of warfarin-associated intracranial hemorrhages, clinical outcomes, and post-intracranial hemorrhage antithrombotic therapy decisions.

We conducted a retrospective review of the AMS database. Eligible patients were taking warfarin at the time of intracranial hemorrhage, had antithrombotic treatment managed by the AMS for at least three-month post-hemorrhage, and were 18 years or greater. Medical records were reviewed from both the AMS and the hospitals where the patients presented. The protocol was approved by the Health Research Ethics Board at the University of Alberta (Pro00089835).

We identified 13 cases between April 2005 and October 2018. Four patients had a mechanical aortic valve, six patients had a mechanical mitral valve, and three patients had both

From the Faculty of Pharmacy and Pharmaceutical Sciences, University of Alberta, Alberta, Canada (AW); Prince of Wales Clinical School, Department of Medicine, University of New South Wales, Sydney, Australia (KB); Division of Neurology, Department of Medicine, University of Alberta, Alberta, Canada (KB); Department of Pharmacy, Alberta Health Services, Alberta, Canada (MH, KS); Division of Cardiology, Department of Medicine, University of Alberta, Alberta, Canada (TJB)

Received September 11, 2019. Final Revisions Submitted November 15, 2019. Date of Acceptance November 25, 2019.

Correspondence to: Dr. Tammy J. Bungard, Division of Cardiology, Department of Medicine, University of Alberta, 362 Heritage Medical Research Centre, Edmonton, Alberta T6G 2S2, Canada. Phone: 780-492-3431. Email: tammy.bungard@ualberta.ca 
valves (Table 1). All known valve types were bileaflet $(N=9)$. The median age was 59 years (interquartile range (IQR): 15 years), many had atrial fibrillation/flutter (AF, $N=8)$, half had a history of hypertension $(N=7)$, few had chronic kidney disease $(N=3)$, and none had hepatic disease. Prior to presentation with intracranial hemorrhage, the target INR range was 2.0-3.0 for 3 patients ( 2 aortic, 1 mitral MHV), 2.5-3.5 for 10 patients ( 1 aortic, 6 mitral, 3 with both MHVs), and concomitant acetyl salicylic acid (ASA) administered in 8 patients ( 3 aortic, 4 mitral, 1 with both MHVs; none had a history of coronary artery disease, previous acute coronary syndrome or myocardial infarction). The median time to presentation from MHV implant to intracranial hemorrhage presentation was 9 years, 3.5 months (IQR: 12 years, 5 months).

The median Glasgow Coma Scale (GCS) score at the time of presentation with intracranial hemorrhage was 15 (IQR: 1.25). Of the 13 incident cases, 4 were intracerebral hemorrhages, 8 were subdural hemorrhages ( 4 were traumatic, 1 had subarachnoid bleeding), and 1 was a traumatic subarachnoid hemorrhage. The initial INRs varied, ranging from 1.4 to 8.4. Based on the target INRs prior to the inciting event, three INRs were subtherapeutic, seven were within range, and six were supratherapeutic upon presentation to a hospital. Anticoagulant reversal was attempted in 12 patients, and the INR was successfully reversed in less than 4 hours $(N=3)$ and greater than 4 hours $(N=9)$ irrespective of the reversal strategy used (e.g., prothrombin complex concentrate (PCC), vitamin K, fresh frozen plasma (FFP)). There were three recurrent hemorrhages in two patients. The median time to recurrent intracranial hemorrhage events was 3 months (IQR: 9 months). Following the initial computerized tomography (CT) scan, surgical procedures were completed without further complications $(N=7)$.

All patients who survived the index event were reinitiated on therapeutic anticoagulation with either warfarin and intravenous heparin $(N=4)$ or warfarin alone $(N=11)$ (median time to reinitiation: 17 days, IQR: 21.5 days). In trauma-related and intracerebral hemorrhages, the median time to reinitiation was 29 (IQR: 17 days) and 14 days (IQR: 28 days), respectively. Only one patient received pharmacologic venous thromboembolism prophylaxis before therapeutic anticoagulation.

Post-intracranial hemorrhage, the INR target range was modified in nine patients (INR target lowered $(N=4$, one aortic, two mitral, one with both MHVs), lowered and narrowed $(N=3$, two aortic, one mitral MHV), or narrowed $(N=2$, one mitral, one with both MHVs)). $75 \%$ and $56 \%$ of patients with the same or modified INR post-intracranial hemorrhage had AF, respectively. Of the eight patients on concomitant ASA and warfarin at the time of presentation with intracranial hemorrhage, four (one aortic, three mitral MHVs) were restarted post-intracranial hemorrhage (median time: 9.5 days, IQR: 10 days). Upon discharge, the median total follow-up by the AMS post-intracranial hemorrhage was 3 years 1 month (IQR: 5 years, 1 month).

Overall, this case series of 13 patients with MHVs who developed anticoagulant-associated intracranial hemorrhage was heterogeneous in terms of type of intracranial hemorrhage, INR reversal strategy employed, timing of warfarin reinitiation, post-intracranial hemorrhage INR target, and concomitant ASA therapy. There were no obvious practice patterns with respect to
MHV location, type and location of intracranial hemorrhage, hematoma expansion, or AF history and the timing of anticoagulant reinitiation, post-intracranial hemorrhage INR targets, or ASA reinitiation. Despite the heterogeneity, the majority of patients presenting with a GCS of 15 recovered without subsequent hemorrhagic or thromboembolic complications.

The majority of our cases presented with either therapeutic $(N=7)$ or supratherapeutic $(N=6)$ INRs, consistent with the literature reporting that a majority (50-90\%) of warfarinassociated intracranial hemorrhage occurs within the target range. ${ }^{5}$ Within our cases, reversal strategies were variable and generally ineffective in terms of rapid INR reversal; only three patients achieved INR reversal within 4 hours of diagnosis. This may reflect changes in practice patterns over the course of this case series. Over time, PCC has replaced FFP and vitamin K alone as the recommended therapy in patients with warfarinassociated intracranial hemorrhage. However, even PCC appeared to take time to reverse INR in our case series. This is also consistent with published data where INR reversal with PCC can take as long as 1610 minutes. $^{6}$

In our case series, the anticoagulation regimen (warfarin monotherapy, warfarin with intravenous heparin bridging (with and without bolus)), and time to restart was widespread (range: 3-46 days). There are no randomized studies of reinitiation of anticoagulation after intracranial hemorrhage in patients with MHVs, and observational data are fragmented. Specifically, Chandra et al. reported that therapeutic heparin can be restarted 3 days following intracranial hemorrhage and switched to warfarin at day 7 without concerns for major bleeding, ${ }^{4}$ while a more recent study by Kuramatsu et al. reported an increased incidence in hemorrhagic complications when therapeutic anticoagulation was restarted in less than two weeks. ${ }^{3}$ Patients in the case series by Babikian et al. resumed warfarin after a mean interval of 19 days, and thromboembolic events were not observed. ${ }^{7}$ Irrespective of the time to therapeutic anticoagulation restart, the outcomes of patients in our case series following intracranial hemorrhage were positive. Patients neither experienced a thromboembolic complication, nor an acute rebleed. The recurrent bleeding rate was low $(N=2)$ and occurred months following reanticoagulation (median: 3 months, IQR: 9 months). These findings were similar to a study by Phan et al. where patients at high embolic risk had a low probability of embolic events when warfarin therapy was discontinued for one to two weeks and intracranial hemorrhage recurrence was uncommon. ${ }^{8}$ Our study suggests that the likelihood of both early recurrent hemorrhage and thromboembolic events is lower than previously suspected (the thrombogenicity of more modern MHVs may be less than their historical counterparts). It is therefore reasonable to reverse the therapeutic coagulopathy in cases of intracranial bleeding and restart once considered stable by the management team.

Unlike previous case series, we assessed INR target modifications post-intracranial hemorrhage $(N=9)$. The majority of our patients had INR target ranges lowered $(N=4)$, lowered and narrowed $(N=3)$, or narrowed $(N=2)$. A lower INR target has been suggested after anticoagulant-associated intracranial hemorrhage, although this is largely based on opinion rather than evidence. ${ }^{9}$ One other case series indicated that $69 \%$ of patients had a lower INR target post-intracranial hemorrhage (pre-intracranial hemorrhage INR target and subsequent 


\begin{tabular}{|c|c|c|c|c|c|c|c|c|c|c|c|c|c|c|c|c|c|}
\hline $\begin{array}{l}\text { Age, } \\
\mathbf{M} / \mathbf{F}\end{array}$ & \begin{tabular}{|l} 
Valve \\
location \\
and type
\end{tabular} & $\begin{array}{c}\text { Prebleed } \\
\text { INR } \\
\text { target }\end{array}$ & $\begin{array}{l}\text { ASA on } \\
\text { presentation }\end{array}$ & $\begin{array}{l}\text { Time from } \\
\text { MHV } \\
\text { implant to } \\
\text { intracranial } \\
\text { hemorrhage } \\
\end{array}$ & $\begin{array}{l}\text { Type and } \\
\text { location of } \\
\text { hemorrhage; } \\
\text { etiology }\end{array}$ & Expansion & $\begin{array}{c}\text { GCS at } \\
\text { presentation }\end{array}$ & $\begin{array}{c}\text { INR at } \\
\text { presentation }\end{array}$ & $\begin{array}{l}\text { Initial INR } \\
\text { reversal } \\
\text { strategy }\end{array}$ & $\begin{array}{l}\text { Time to INR } \\
\text { reversal } \\
\text { (INR }<1.5 \text { ) }\end{array}$ & Neurosurgery & $\begin{array}{c}\text { Days to } \\
\text { ASA } \\
\text { reinitiation }\end{array}$ & $\begin{array}{c}\text { Days to } \\
\text { OAC } \\
\text { reinitiation }\end{array}$ & \begin{tabular}{|l} 
Anticoagulation \\
regimen \\
restarted
\end{tabular} & $\begin{array}{l}\text { Postbleed } \\
\text { INR target }\end{array}$ & $\begin{array}{l}\text { Total follow-up } \\
\text { posthemorrhage }\end{array}$ & $\begin{array}{l}\text { Pertinent } \\
\text { medical } \\
\text { history }\end{array}$ \\
\hline $53 \mathrm{M}$ & \begin{tabular}{|l|} 
Mitral, \\
unknown*
\end{tabular} & $2.0-3.0$ & No & 18 years & $\begin{array}{l}\text { Intracerebral, } \\
\text { brainstem/ } \\
\text { cerebellum; } \\
\text { cavernoma }\end{array}$ & Yes & 15 & 2.4 & \begin{tabular}{|l|} 
Octaplex \\
$1000 \mathrm{IU}$, \\
then vitamin \\
K $10 \mathrm{mg}$ \\
$\mathrm{PO} \times 3$ days \\
\end{tabular} & 8 hours & $\begin{array}{l}\text { Craniotomy and } \\
\text { resection of } \\
\text { cavernous } \\
\text { malformation }\end{array}$ & N/A & 46 & $\begin{array}{l}\text { Warfarin and } \\
\text { heparin IV }\end{array}$ & $2.0-3.0$ & 1 year, 1 month & $\begin{array}{l}\text { AF, history } \\
\text { of previous } \\
\text { pontine } \\
\text { hemorrhage }\end{array}$ \\
\hline $35 \mathrm{M}$ & Aortic, On-X & $2.0-3.0$ & Yes & 17 days & $\begin{array}{l}\text { Intracerebral, } \\
\text { lobar, with } \\
\text { intraventricular } \\
\text { extension }\end{array}$ & No & 13 & 2.7 & $\begin{array}{l}\text { Octaplex } \\
1000 \mathrm{IU}\end{array}$ & 24 hours & $\begin{array}{l}\text { Craniotomy, } \\
\text { evacuation of } \\
\text { intraparenchymal } \\
\text { hematoma }\end{array}$ & 7 & 14 & $\begin{array}{l}\text { Warfarin and } \\
\text { heparin IV }\end{array}$ & $1.8-2.2$ & 1 year & Atrial flutter \\
\hline $47 \mathrm{~F}$ & $\begin{array}{l}\text { Mitral, } \\
\text { unknown* }\end{array}$ & $2.5-3.5$ & Yes & $\begin{array}{l}8 \text { years, } \\
4 \text { months }\end{array}$ & $\begin{array}{l}\text { Intracerebral, } \\
\text { lobar, with } \\
\text { intraventricular } \\
\text { extension }\end{array}$ & No & 15 & 8.5 & \begin{tabular}{|l|} 
Vitamin $\mathrm{K}$ \\
$10 \mathrm{mg} \mathrm{IV} \times 1$
\end{tabular} & 3 hours & $\begin{array}{l}\text { Craniotomy and } \\
\text { evacuation of } \\
\text { hemorrhage }\end{array}$ & No & 5 & $\begin{array}{l}\text { Warfarin and } \\
\text { heparin IV }\end{array}$ & $2.0-3.0$ & $\begin{array}{l}13 \text { years, } \\
8 \text { months }\end{array}$ & \multirow[t]{2}{*}{ Ex-smoker } \\
\hline $47 \mathrm{~F}$ & $\begin{array}{l}\text { Mitral, } \\
\text { unknown* }\end{array}$ & $2.5-3.0$ & Yes & $\begin{array}{l}8 \text { years, } \\
6 \text { months }\end{array}$ & $\begin{array}{l}\text { Intracerebral, } \\
\text { lobar, and } \\
\text { cerebellar } \\
\text { hemisphere, } \\
\text { with } \\
\text { intraventricular } \\
\text { extension }\end{array}$ & No & 15 & 6.9 & $\mathrm{FFP} \times 4$ & 10 hours & $\begin{array}{l}\text { Craniotomy and } \\
\text { evacuation of } \\
\text { hemorrthage }\end{array}$ & 30 & 10 & Warfarin & $2.5-3.0$ & $\begin{array}{l}13 \text { years, } \\
6 \text { months }\end{array}$ & \\
\hline $50 \mathrm{M}$ & Mitral, On-X & $2.5-3.5$ & Yes & $\begin{array}{l}1 \text { year, } \\
7 \text { months }\end{array}$ & $\begin{array}{l}\text { Intracerebral, } \\
\text { deep, with } \\
\text { intraventricular } \\
\text { extension }\end{array}$ & No & 8 & 2.9 & \begin{tabular}{|l|} 
Octaplex \\
$1000 \mathrm{IU}$, \\
vitamin $\mathrm{K}$ \\
$10 \mathrm{mg} \mathrm{IV} \times 3$ \\
\end{tabular} & 2 hours & None & NR & 38 & Warfarin & $2.5-3.0$ & 1 year, 4 months & $\begin{array}{l}\text { HTN, CKD } \\
\text { (Stage 2) }\end{array}$ \\
\hline $68 \mathrm{~F}$ & $\begin{array}{l}\text { Mitral and } \\
\text { aortic, } \\
\text { unknown* }\end{array}$ & $2.5-3.5$ & No & 20 years & $\begin{array}{l}\text { Subdural } \\
\text { (acute); trauma }\end{array}$ & No & 15 & 3.4 & Not reversed & $\begin{array}{l}\text { Patient } \\
\text { discharged } \\
\text { from } \\
\text { Emergency } \\
\text { Department }\end{array}$ & None & N/A & 14 & Warfarin & $2.5-3.0$ & 6 years, 3 months & $\begin{array}{l}\text { AF, history } \\
\text { of } \\
\text { posttraumatic } \\
\text { severe } \\
\text { intracerebral } \\
\text { hemorrhage }\end{array}$ \\
\hline $66 \mathrm{M}$ & \begin{tabular}{|l} 
Aortic, St. \\
Jude
\end{tabular} & $2.5-3.5$ & Yes & $\begin{array}{l}12 \text { years, } \\
4 \text { months }\end{array}$ & $\begin{array}{l}\text { Subdural } \\
\text { (bilateral, } \\
\text { acute); trauma }\end{array}$ & No & 14 & 2.5 & FFP $\times 1$ & 24 hours & $\begin{array}{l}\text { Burr hole } \\
\text { drainage of } \\
\text { hematomas }\end{array}$ & No & $38 * *$ & Warfarin & $2.0-3.0$ & 2 years, 1 month & HTN \\
\hline $65 \mathrm{M}$ & \begin{tabular}{|l|} 
Aortic, St. \\
Jude
\end{tabular} & $2.5-3.5$ & No & $\begin{array}{l}9 \text { years, } \\
7 \text { months }\end{array}$ & $\begin{array}{l}\text { Subdural } \\
\text { (acute); trauma }\end{array}$ & No & 15 & 2.9 & \begin{tabular}{|l|} 
Octaplex \\
$1000 \mathrm{IU}$, \\
vitamin $\mathrm{K}$ \\
$10 \mathrm{mg} \mathrm{IV} \times 1$ \\
\end{tabular} & 13.5 hours & None & N/A & 17 & Warfarin & $2.5-3.5$ & 3 years, 1 month & \multirow[t]{3}{*}{$\begin{array}{l}\text { History of } \\
\text { alcohol use } \\
\text { disorder, } \\
\text { active } \\
\text { smoker }\end{array}$} \\
\hline $67 \mathrm{M}$ & \begin{tabular}{|l|} 
Aortic, St. \\
Jude
\end{tabular} & $2.5-3.5$ & No & $\begin{array}{l}11 \text { years, } \\
3 \text { months }\end{array}$ & $\begin{array}{l}\text { Subdural } \\
\text { (recurrent), } \\
\text { subarachnoid, } \\
\text { intracerebral, } \\
\text { intraventricular } \\
\text { with extension; } \\
\text { trauma }\end{array}$ & Yes & $14-15$ & 1.4 & \begin{tabular}{|l|} 
Vitamin $\mathrm{K}$ \\
$5 \mathrm{mg} \mathrm{IV} \times 1$
\end{tabular} & 20 hours & None & N/A & 7 & $\begin{array}{l}\text { Warfarin and } \\
\text { heparin IV }\end{array}$ & $2.5-3.5$ & 5 months & \\
\hline $67 \mathrm{M}$ & \begin{tabular}{|l} 
Aortic, St. \\
Jude
\end{tabular} & $2.5-3.5$ & No & $\begin{array}{l}11 \text { years, } \\
6 \text { months }\end{array}$ & $\begin{array}{l}\text { Subdural, } \\
\text { subarachnoid; } \\
\text { trauma }\end{array}$ & No & 3 & 4 & Not reversed & $\begin{array}{l}\text { Died within } \\
72 \text { hours of } \\
\text { presentation to } \\
\text { hospital }\end{array}$ & None & N/A & NR & N/A & N/A & N/A & \\
\hline $50 \mathrm{~F}$ & \begin{tabular}{|l} 
Mitral, St. \\
Jude
\end{tabular} & $2.5-3.5$ & Yes & 13 days & $\begin{array}{l}\text { Subdural (acute } \\
\text { and chronic) }\end{array}$ & Yes & 12 & 1.7 & \begin{tabular}{|l|} 
Octaplex \\
$1000 \mathrm{IU}$, \\
vitamin $\mathrm{K}$ \\
$10 \mathrm{mg} \mathrm{IV} \times 1$
\end{tabular} & 10.5 hours & None & 5 & 30 & Warfarin & $1.7-2.0$ & 6 years, 1 month & AF, HTN \\
\hline
\end{tabular}


Table 1. Continued

\begin{tabular}{|c|c|c|c|c|c|c|c|c|c|c|c|c|c|c|c|c|c|}
\hline $\begin{array}{l}\text { Age, } \\
\text { M/F }\end{array}$ & $\begin{array}{l}\text { Valve } \\
\text { location } \\
\text { and type }\end{array}$ & $\begin{array}{c}\text { Prebleed } \\
\text { INR } \\
\text { target }\end{array}$ & $\begin{array}{l}\text { ASA on } \\
\text { presentation }\end{array}$ & $\begin{array}{l}\text { Time from } \\
\text { MHV } \\
\text { implant to } \\
\text { intracranial } \\
\text { hemorrhage }\end{array}$ & $\begin{array}{l}\text { Type and } \\
\text { location of } \\
\text { hemorrhage; } \\
\text { etiology }\end{array}$ & Expansion & $\begin{array}{c}\text { GCS at } \\
\text { presentation }\end{array}$ & $\begin{array}{c}\text { INR at } \\
\text { presentation }\end{array}$ & $\begin{array}{l}\text { Initial INR } \\
\text { reversal } \\
\text { strategy }\end{array}$ & \begin{tabular}{|l} 
Time to INR \\
reversal \\
$($ INR $<1.5)$
\end{tabular} & Neurosurgery & $\begin{array}{c}\text { Days to } \\
\text { ASA } \\
\text { reinitiation }\end{array}$ & $\begin{array}{c}\text { Days to } \\
\text { OAC } \\
\text { reinitiation }\end{array}$ & $\begin{array}{l}\text { Anticoagulation } \\
\text { regimen } \\
\text { restarted }\end{array}$ & $\begin{array}{l}\text { Postbleed } \\
\text { INR target }\end{array}$ & $\begin{array}{c}\text { Total follow-up } \\
\text { posthemorrhage }\end{array}$ & \begin{tabular}{|l} 
Pertinent \\
medical \\
history
\end{tabular} \\
\hline $52 \mathrm{M}$ & Aortic, On-X & $2.0-3.0$ & Yes & 8 months & $\begin{array}{l}\begin{array}{l}\text { Subdural } \\
\text { (chronic) }\end{array} \\
\text { (c) }\end{array}$ & Yes & 15 & 3.2 & \begin{tabular}{|l|} 
Vitamin $\mathrm{K}$ \\
$2 \mathrm{mg} \mathrm{PO} \times 1$
\end{tabular} & 2 hours & \begin{tabular}{|l|} 
Burr hole \\
drainage of \\
hematoma
\end{tabular} & NR & 18 & Warfarin & $1.8-2.5$ & \begin{tabular}{|l|}
2 years, \\
11 months
\end{tabular} & \\
\hline $78 \mathrm{~F}$ & $\begin{array}{l}\text { Mitral, St. } \\
\text { Jude }\end{array}$ & $2.5-3.5$ & No & 8 years & $\begin{array}{l}\text { Subdural } \\
\text { (bilateral, } \\
\text { chronic) }\end{array}$ & No & 15 & 5.4 & Not reversed & \begin{tabular}{|l|} 
Patient \\
discharged \\
from \\
Emergency \\
Department
\end{tabular} & None & N/A & 8 & Warfarin & $2.0-3.0$ & 6 years & \begin{tabular}{|l} 
AF, HTN, \\
CKD (Stage \\
3B), history \\
of left \\
parietal \\
infarct \\
\end{tabular} \\
\hline $63 \mathrm{M}$ & $\begin{array}{l}\text { Mitral and } \\
\text { oortic, St. } \\
\text { Jude }\end{array}$ & $2.5-3.5$ & No & 9 years & $\begin{array}{l}\text { Subdural } \\
\text { (multiple; acute } \\
\text { and chronic); } \\
\text { trauma }\end{array}$ & No & 15 & 1.5 & $\operatorname{FFP} \times 1$ & 18 hours & $\begin{array}{l}\text { Burr hole } \\
\text { evacuation of } \\
\text { hematoma }\end{array}$ & N/A & 29 & Warfarin & $2.0-3.0$ & 6 years, 4 months & $\begin{array}{l}\text { AF, HTN, } \\
\text { CKD (Stage } \\
\text { 3A), active } \\
\text { smoker }\end{array}$ \\
\hline $59 \mathrm{~F}$ & $\begin{array}{l}\text { Mitral, St. } \\
\text { Jude }\end{array}$ & $2.5-3.5$ & Yes & 14 years & $\begin{array}{l}\text { Subdural, } \\
\text { Subarachnoid }\end{array}$ & No & 15 & 8.4 & $\begin{array}{l}\text { Octaplex } \\
1000 \mathrm{IU} \times 3 \text {, } \\
\text { platelets } \times 1 \text {, } \\
\text { vitamin } \mathrm{K} \\
10 \mathrm{mg} \mathrm{IV} \times 3\end{array}$ & 4 hours & None & 12 & 31 & Warfarin & $2.5-3.5$ & 1 year & AF, HTN \\
\hline $60 \mathrm{~F}$ & $\begin{array}{l}\text { Mitral and } \\
\text { aortic, } \\
\text { unknown* }\end{array}$ & $2.5-3.5$ & Yes & 16 years & $\begin{array}{l}\text { Subarachnoid; } \\
\text { trauma }\end{array}$ & No & 15 & 3.6 & Not reversed & N/A & None & NR & 3 & Warfarin & $2.5-3.5$ & \begin{tabular}{|l|}
6 years, \\
11 months
\end{tabular} & \begin{tabular}{|l} 
AF, HTN, \\
history of \\
UGIB \\
secondary to \\
esophagitis \\
3 years prior \\
to initial \\
intracranial \\
hemorrhage \\
event
\end{tabular} \\
\hline
\end{tabular}

Shaded rows indicate patients who had recurrent intracranial hemorrhage.

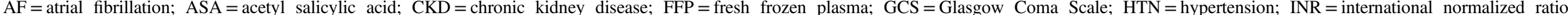

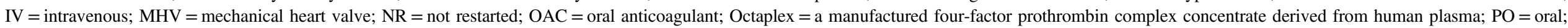
UGIB = upper gastrointestinal bleed.

*Valve type not documented in charts reviewed.

**Patient was initiated on low molecular weight heparin (LMWH) for venous thromboembolism (VTE) prophylaxis 3 days after event and continued until OAC initiation. 
correlation with patient outcomes were not documented). ${ }^{10}$ Our case series reports that both patients with the same or modified INR target post-intracranial hemorrhage did not result in any subsequent thromboembolic events. Similarly, patients reinitiated on concomitant ASA and warfarin therapy or reduced to warfarin monotherapy did not experience any subsequent rebleeding or thromboembolic event in follow-up. While narrowing the target range may be more achievable in an AMS, this approach likely mandates more frequent INR testing and mitigates larger fluctuations in sub/supratherapeutic INRs when compared to a wider target range.

Limitations of this case series are its small sample size and retrospective design. Data collection based on chart review limits the precision of the results presented (e.g., variability in number and timing of follow-up INR testing and CT scans).

In conclusion, antithrombotic management following intracranial hemorrhage among our cases was variable, and neither correlated with the type, location, or etiology of bleed, nor the valve type and associated thromboembolic risk. Diligent assessment and management of INR may improve long-term outcomes more than timing of warfarin restart and/or modified target INR.

\section{CONFLICT OF INTEREST}

$\mathrm{AW}, \mathrm{MH}$, and $\mathrm{KS}$ have nothing to disclose.

$\mathrm{KB}$ has received speaker honoraria, served on advisory boards, and received research grant support from BoehringerIngelheim, Bayer, Pfizer, and Servier.

TJB has received speaker honoraria from Bayer and unrestricted research grants from Bayer and Pfizer.

\section{Statement of Authorship}

AW contributed to the concept and design, analysis, or interpretation of the data, critical revision with intellectual content, and final approval of the version to be published.

$\mathrm{KB}$ contributed to the design, analysis, or interpretation of the data, critical revision with intellectual content, and final approval of the version to be published.
$\mathrm{MH}$ contributed to the interpretation of the data, critical revision with intellectual content, and final approval of the version to be published.

KS contributed to the concept, interpretation of the data, critical revision with intellectual content, and final approval of the version to be published.

TJB contributed to the concept and design, analysis, or interpretation of the data, critical revision with intellectual content, and final approval of the version to be published.

\section{REFERENCES}

1. Eikelboom JW, Connolly SJ, Brueckmann M, et al. Dabigatran versus warfarin in patients with mechanical heart valves. N Engl J Med. 2013;369(13):1206-14.

2. Puskas JD, Gerdisch M, Nichols D, et al. Anticoagulation and antiplatelet strategies after On-X mechanical aortic valve replacement. J Am Coll Cardiol. 2018;71(24):2717-26.

3. Kuramatsu JB, Sembill JA, Gerner ST, et al. Management of therapeutic anticoagulation in patients with intracerebral haemorrhage and mechanical heart valves. Eur Heart J. 2018;39(19):1709-23.

4. Chandra D, Gupta A, Grover V, et al. When should you restart anticoagulation in patients who suffer an intracranial bleed who also have a prosthetic valve? Interact Cardiovasc Thorac Surg. 2013;16(4):520-3.

5. Yung D, Kapral MK, Asllani E, et al. Reinitiation of anticoagulation after warfarin-associated intracranial hemorrhage and mortality risk: the Best Practice for Reinitiating Anticoagulant Therapy After Intracranial Bleeding (BRAIN) study. Can J Cardiol. 2012;28(1):33-9.

6. Steiner T, Poli S, Griebe M, et al. Fresh frozen plasma versus prothrombin complex concentrate in patients with intracranial haemorrhage related to vitamin $\mathrm{K}$ antagonists (INCH): a randomised trial. Lancet Neurol. 2016;15(6):566-73.

7. Babikian VL, Kase CS, Pessin MS, et al. Resumption of anticoagulation after intracranial bleeding in patients with prosthetic heart valves. Stroke. 1988;19(3):407-8.

8. Phan TG, Koh M, Wijdicks EF. Safety of discontinuation of anticoagulation in patients with intracranial hemorrhage at high thromboembolic risk. Arch Neurol. 2000;57(12):1710-3.

9. Li YG, Lip GYH. Anticoagulation resumption after intracerebral hemorrhage. Curr Atheroscler Rep. 2018;20(7):32.

10. Butler AC, Tait RC. Restarting anticoagulation in prosthetic heart valve patients after intracranial haemorrhage: a 2-year follow-up. Br J Haematol. 1998;103(4):1064-6. 